\title{
Particle Swarm Optimization-Based Rectangular Microstrip Antenna Designing
}

\author{
Hadi Sadoghi Yazdi ${ }^{1}$, Mehri Sadoghi Yazdi ${ }^{2}$
}

\begin{abstract}
In this paper, a new system is proposed for microstrip antenna (MSA) designing. Two main stages need for designing procedure. Firstly, modeling of rectangular MSA is performed using some nonlinear regression methods. Then an inverse modeling is done using particle swarm optimization (PSO) algorithm with some constraints. Result includes a suitable system with bandwidth as an input and dimensions width, frequency range, length over a ground plane with a substrate thickness, dielectric loss tangent and electrical thickness as an output.
\end{abstract}

Index Terms - Particle swarm optimization, Microstrip antennas, Nonlinear regression

\section{INTRODUCTION}

Designing procedure can be performed based on modeling and inverse system. In this way, some experimental samples are captured from real system then it is modeled to form of a function $y=f\left(x_{1}, x_{2}, \ldots, x_{n}\right)$ where output of system is $y$ and input features or parameters are $x_{1}, x_{2} \ldots, x_{n}$. But in designing procedure we need suitable input parameters $\left(x_{1}, x_{2}, \ldots, x_{n}\right)$ for receiving to desired $(y)$, this step require an inverse modeling procedure. Main target in this paper is designing of rectangular microstrip antenna which after its modeling, bandwidth can be calculate using mathematical nonlinear model versus five input parameters include dimensions width, frequency range, length over a ground plane with a substrate thickness and dielectric loss tangent and electrical thickness. Then a artificial search algorithm is used for finding inverse model ( Fig 1) that bandwidth is desire and dimensions width, frequency range, length over a ground plane with a substrate thickness and dielectric loss tangent and electrical thickness as outputs.

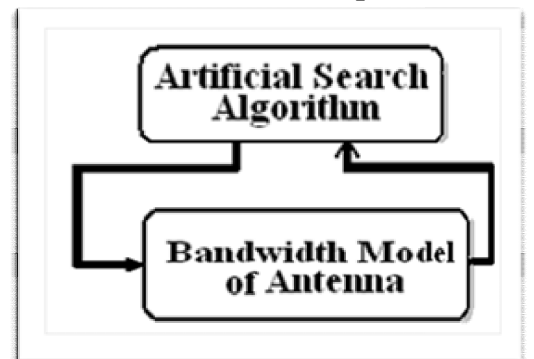

Fig. 1 Searching problem of optimum input parameters for obtaining desired bandwidth which is appeared in the future work

The used artificial search algorithm is swarm intelligent

Hadi Sadoghi Yazdi is with the Computer Department, Ferdowsi University of Mashhad, Mashhad, Iran

Mehri Sadoghi Yazdi is with the Department of Computer engineering, Faculty of Electrical and Computer Engineering, Shahid Beheshti University of Tehran, Iran search. Kennedy and Eberhart [1,2] introduced the Particle Swarm Optimization (PSO) method based on the simulation of bird flocking and fish schooling. PSO is a high performance optimizer that controls several highly desirable attributes, including the fact that the basic algorithm is very easy to comprehend and implement. It is similar in some ways to evolutionary algorithms, but needs less computation and generally fewer lines of code. This algorithm can optimize problem that requires the simultaneous optimization of $\mathrm{N}$ variables. It has attained its popularity due to a broad range of useful applications in such diverse areas as image compression [3], power system [4], phased array synthesis [5], RF circuit design [6], and digital filter design [7].

\section{A. Scope of MSA}

In recent years, the current trend in commercial and military communication systems has been to develop low cost, minimal weight, low profile planar configuration antennas ([8], [9] and [10]) that are capable of maintaining high performance over a large spectrum of frequencies. This technological trend has focused much effort into the design of microstrip (patch) antennas.

The concept of Microstrip antenna though introduced in the early 1950's in USA by Deschamps and in France by Gutton and Baissinot, it was in the 1970's only that with the advent of printed-circuit technology [11, 12], some serious advancements in this research area had begun resulting in the development of first practical antennas.

A Microstrip device in its simplest form is a sandwich of two parallel conducting layers separated by a single thin dielectric substrate. The upper conductor is a thin metallic patch (usually Copper or Gold), which is a small fraction of a wavelength [8]. The lower conductor is a ground plane which should be infinite theoretically. The patch and ground-plane are separated by a dielectric substrate which is usually non-magnetic. The patch can assume any shape, be it rectangular, circular, triangular, elliptical, helical, circular ring, etc. The variety in design that is possible with Microstrip antennas probably exceeds that of any other type of antenna element. Microstrip antennas are used where size, weight, cost, better performance, compatibility with microwave and millimeter wave integrated circuits (MMICs), robustness, ability to conform to planar and non-planar surfaces, etc. are required $[11,13]$.

Bandwidth and efficiency of a Microstrip antenna depends upon patch size, shape, substrate thickness, dielectric constant of substrate, feed point type and its location, etc. For good antenna performance, a thick dielectric substrate having a low dielectric constant is desirable for higher bandwidth, better efficiency and better radiation, leading to a larger 
antenna size. Designing a compact antenna requires higher dielectric constant, leading to narrower bandwidth, lesser efficiency and higher loss tangents (dissipation factors) [11].

In MSA designs, it is important to determine the bandwidth of the antenna accurately because the bandwidth is a critical parameter of a MSA. Several techniques [14, 15, 16], varying in accuracy and computational effort, have been proposed and used to calculate the bandwidth of a rectangular MSA, as this is one of the most popular and convenient shapes. The analytical techniques use simplifying physical assumptions, but generally offer simple and analytical solutions that are well-suited for an understanding of the physical phenomena and for antenna computer-aided design. These analytical techniques are known as transmission-line models and cavity models.

However, these techniques are not suitable for many structures, in particular, if the thickness of the substrate is not very thin. Most of the limitations of analytical techniques can be overcome by using the numerical techniques. The numerical techniques are based on an electromagnetic boundary problem, which leads to an expression as an integral equation, using proper Green functions, either in the spectral domain, (the SDA method), or directly in the space domain, using the method of moments. Without any initial assumption, the choice of test functions and the path integration appear to be more critical during the final, numerical solution.

Exact mathematical formulations in rigorous numerical methods involve extensive numerical procedures, resulting in round-off errors, and may also need final experimental adjustments to the theoretical results. These methods also suffer from the fact that any change in the geometry (patch shape, feeding method, addition of a cover layer, etc.) requires the development of a new solution. Furthermore, most of the previous theoretical and experimental work has been carried out only with electrically thin MSAs, normally of the order of $h / R_{d} \leq 0.14$, where $h$ is the thickness of the dielectric substrate and ${ }^{\lambda} \mathrm{d}$ is the wavelength in the substrate.

Recent interest has developed in radiators etched on electrically thick substrates. This interest is primarily for two major reasons. First, as these antennas are used for applications with increasingly higher operating frequencies, and consequently shorter wavelength, even antennas with physically thin substrates become thick when compared to a certain wavelength. Second, the bandwidth of the rectangular MSA is typically very small for low profile, electrically thin configurations. One of the techniques to increase the bandwidth is to increase the thickness proportionately. The design of MSA elements having wider bandwidth is an area of major interest in MSA technology, particularly in the fields of electronic warfare, communication systems, and wideband radars. Consequently, this problem, particularly the bandwidth aspect, has received considerable attention.

The reminder of paper is organized as follows. In section 2, the bandwidth of an MSA is described, and then in section 3 MSA modeling is presented using nonlinear mathematical model. Section 4 includes the PSO-based designing procedure with a review on Particle Swarm Optimization (PSO) methods and its main parts for designing process.
Experimentations are described in section 5 and finally section 6 draws conclusion of this paper.

\section{BANDWIDTH OF RECTANGULAR MiCROSTRIP ANTENNAS}

The rectangular microstrip antennas are made of a rectangular patch with dimensions width, $\mathrm{W}$ and length, L, over a ground plane with a substrate thickness $h$ and relative dielectric constants er, as given in Fig.2.

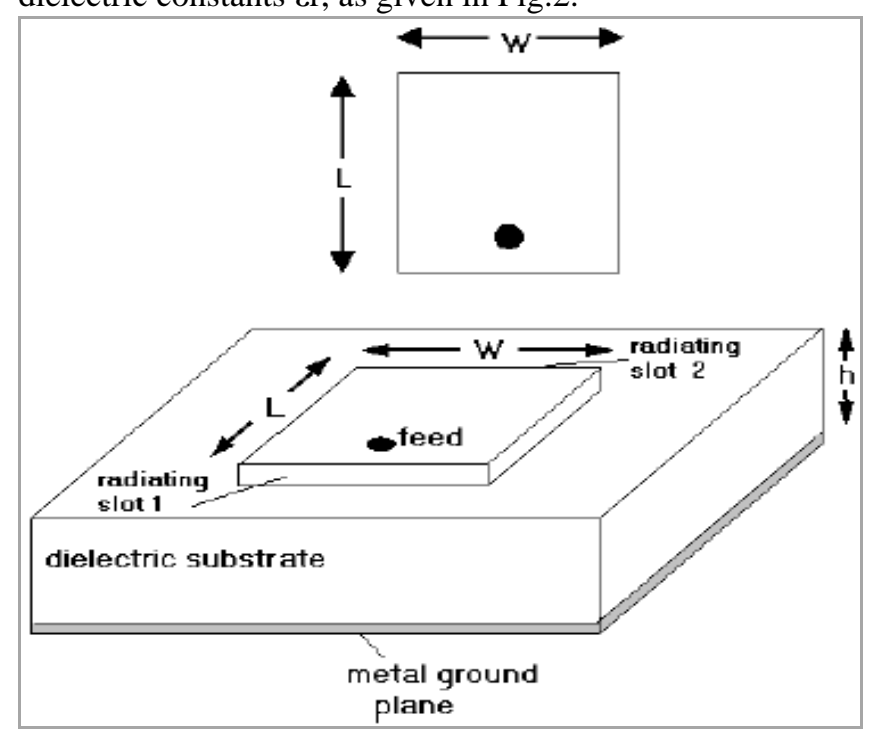

Fig.2. Rectangular microstrip antenna

The bandwidth of this MSA can be determined from the frequency response of its equivalent circuit. For a paralleltype resonance, the bandwidth is expressed as [22]

$$
B W=\frac{2 G}{\left.\omega_{r} \frac{d B}{d \omega}\right|_{\omega_{r}}}
$$

Where $\mathrm{Y}=\mathrm{G}+\mathrm{jB}$ is the input admittance at the angular resonant frequency $\omega_{r}$. For a series-type resonance, $G$ is replaced by $\mathrm{R}$ and $\mathrm{B}$ is replaced by $\mathrm{X}$ in equation (1), where $\mathrm{Z}=\mathrm{R}+\mathrm{jX}$ is the input impedance at resonance. The bandwidth of a MSA can also be expressed as [21]

$$
B W=\frac{s-1}{Q_{T} \sqrt{s}}
$$

Where $s$ is the voltage standing wave ratio (VSWR) and $\mathrm{Q}_{\mathrm{T}}$ is the total quality factor. The total quality factor, $\mathrm{Q}_{\mathrm{T}}$, can be written as

$$
\frac{1}{Q_{T}}=\frac{P_{d}+P_{c}+P_{r}+P_{s}}{\omega_{r} W_{T}}
$$

Where $P_{d}$ is the power lost in the lossy dielectric substrate, $P_{c}$ is the power lost in the imperfect conductor, $P_{r}$ is the power radiated in the space waves, $P_{s}$ is the power radiated in the surface waves, and $W_{T}$ is the total energy stored in the patch at resonance.

The wavelength in the dielectric substrate, $\lambda_{d}$, is given as

$\lambda_{d}=\frac{\lambda_{0}}{\sqrt{\varepsilon_{r}}}=\frac{c}{f_{r} \sqrt{\varepsilon_{r}}}$

$\lambda_{0}$ is the free space wavelength at the resonant frequency $f_{r}$ and $c$ is the velocity of electromagnetic waves in free space. 
In this paper we introduce a novel method to calculate the bandwidth of rectangular MSAs based on Support Vector Regression (SVR). We use the parameters $\mathrm{h}, f_{r}, h / \lambda_{d}, W$ and $\tan \delta$ for computation of Bandwidth.

\section{MODELING OF BANDWIDTH OF RECTANGULAR MICROSTRIP ANTENNAS}

In this work, the SVR was used to compute the bandwidth of electrically thin and thick rectangular MSAs. For the SVR, the inputs are $h, f_{r}, h / \lambda_{d}, W$ and $\tan \delta$, and the output is the measured bandwidths $B W m e$. The training and test data sets used in this paper have been obtained from previous experimental works $[17,18]$, and are given in Table 5 (which is appeared in Appendix). In this paper we used some kernel functions for SVR like polynomial with different degree, radial basis function and linear function. We use hold out and leave one out method for comparing average error of our proposed method with ANFIS (Adaptive Neuro Fuzzy Inference System) and results are shown in Table 1.

Table 1. Errors of SVR and ANFIS for bandwidths for electrically thin and thick rectangular microstrip antenna with leave one out and hold out method

\begin{tabular}{ccccc}
\hline \multirow{2}{*}{ Kernel } & \multicolumn{2}{c}{ Average Error SVR } & \multicolumn{2}{c}{$\begin{array}{c}\text { Average Error } \\
\text { ANFIS }\end{array}$} \\
\cline { 2 - 5 } & $\begin{array}{c}\text { Leave } \\
\text { one out }\end{array}$ & $\begin{array}{c}\text { Hold } \\
\text { out }\end{array}$ & $\begin{array}{c}\text { Leave } \\
\text { one out }\end{array}$ & $\begin{array}{c}\text { Hold } \\
\text { out }\end{array}$ \\
\hline Poly, $\mathrm{p}=2$ & 37.8586 & 0.0787 & 37.2162 & 0.1757 \\
\hline Poly, $\mathrm{p}=3$ & 19.6580 & 0.1431 & 37.2162 & 0.1757 \\
\hline Poly, $\mathrm{p}=4$ & 37.5095 & 0.1998 & 37.2162 & 0.1757 \\
\hline erbf & 28.8429 & 0.0833 & 37.2162 & 0.1757 \\
\hline rbf & 44.8668 & 0.2530 & 37.2162 & 0.1757 \\
\hline Linear & 20.4241 & 0.0612 & 37.2162 & 0.1757 \\
\hline
\end{tabular}

We can see in Table 1 that 'erbf' kernel function has less average error than ANFIS with both evaluation methods.

\section{A. Fine modeling}

As we mentioned before, the support vector machine is an approximate implementation of the method of structural risk minimization. This induction principle is based on the fact that the error rate of a learning machine on test data is bounded by the sum of the training-error and a term that depends on the Vapnik-Chervonenkis (VC) dimension. In this method, optimal hyper plane is determined to guarantee the minimum error for test samples, whereas, neural networks don't guarantee to find optimum hyper plane for test samples. So SVR give us better results relative to ANFIS as shown in section 4. But, some problems in the SVR are exists as follows:

a) Since each sample is one constraint in support vector, increasing training samples is equivalent to increasing number of constraints. In this case solving equations to find optimal hyper plane becomes very hard.

b) Finding suitable kernel for modeling of nonlinear space.
First Problem in the current work and similar works can be easily seen. For example for leave one out train and test procedure, we have 33 training samples and optimization problem must be solved with 33 constraints. The selection of suitable kernel given in the second problem is clear in Table 1 at a glance. So, we propose new version of SVR that works based on divide and conquer principle which can solves two aforementioned problems. Input space is divided to several subspaces and in each subspace a SVR models the data. This work is caused the new generated space has the properties of high dimensional space. A weighting procedure is performed using probability density function of each sub-space and gives portion of each SVR according to generated rules. Then results of weighted SVRs are combined and fitting is performed. We describe presented SVR method in detail in the following steps.

Step 1: In this step, we should divide input training data into $n$ subsets. We can use an algorithm of clustering like fuzzy c-means (FCM) for this grouping. Indeed we assign the weights to any input data using FCM. Fig. 3 shows 3 partition (for example) which FCM has clustered. PDF (Probability Density Function) of each cluster are obtained as shown in the following figure then the corresponding weights of an input data are calculated based on membership values to each partitions (i.e. clusters).

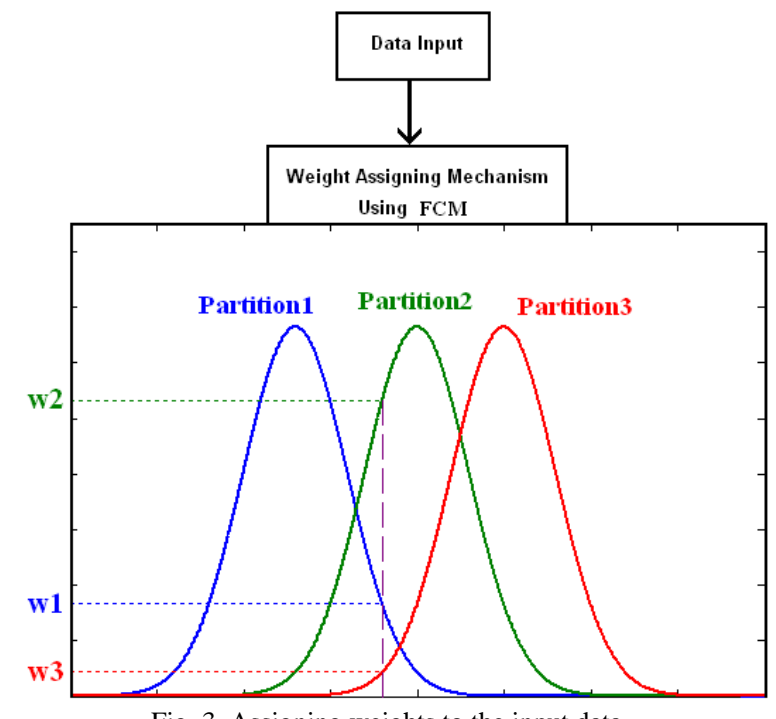

Fig. 3. Assigning weights to the input data

Step 2: Each available subset for any partition are applied for training of each Support Vector Regressor (SVR) like Fig. 4. So, for training samples of partition 1 (as shown in Fig.3) SVR1 is trained (as shown in Fig.4).

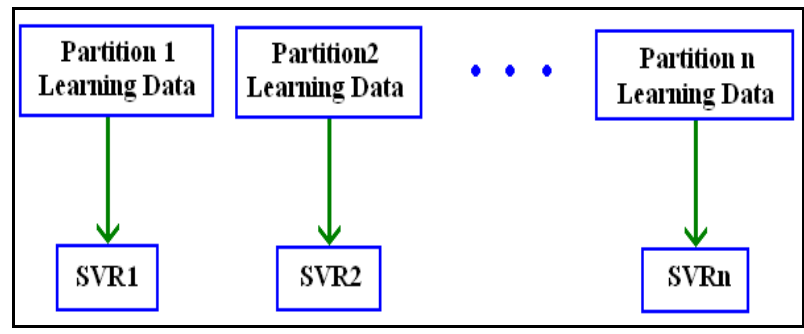

Fig. 4 Applying SVR in each partition 
In this paper we used some kernel functions for SVR like polynomial with different degree, radial basis function and linear and check results for finding best state.

Step 3: Third step is testing procedure. We use leave one out method for computing average error of our proposed method and compare it with ANFIS. In order to calculate the output of the proposed system, we should compute membership values for each test sample $\left(w_{i}\right)$. By applying equation (8) for any test sample, corresponding weights to the test samples are obtained and then we normalize these weights by dividing any weight to the sum of them. At the end, these normalized weights multiplied by each test sample for generating final values. Fig. 5 shows this procedure.

$$
w_{i}=\frac{1}{2 \pi|\tilde{\Sigma}|^{0.5}} \exp \left(-\left(x_{t}-\mu\right) \tilde{\Sigma}^{-1}\left(x_{t}-\mu\right)^{T}\right)
$$

Where $\tilde{\Sigma}=\alpha \sum$ and $\sum$ is covariance samples and $\mu$ is mean value of training data.

In equation (8), $\alpha$ is a variable to control of spreading of each Gaussian distribution that we consider for samples of training set. $x_{t}$ is a test sample. Also $|\cdot|$ denotes to determinant.

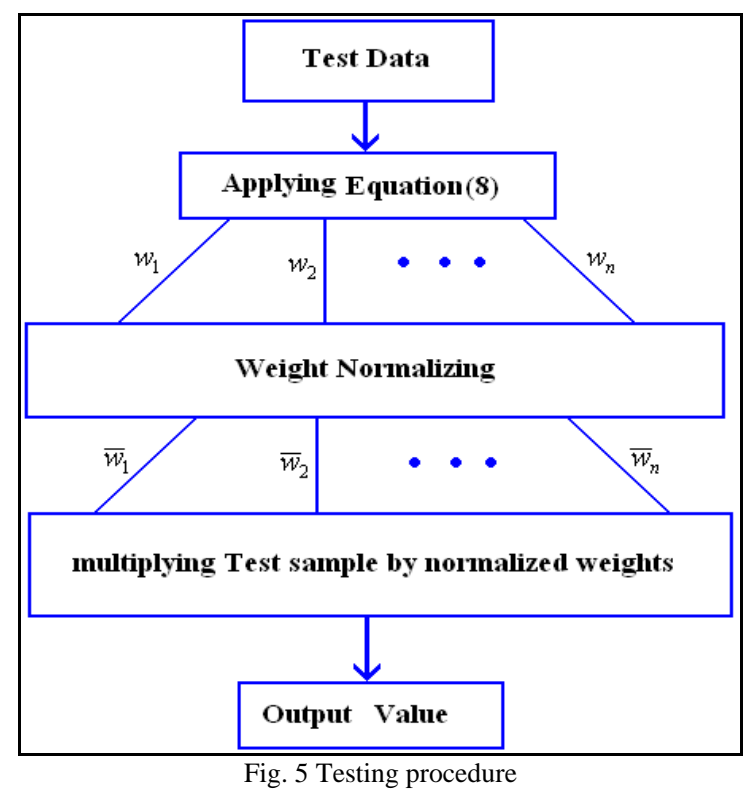

In Fig. 5 output value is computed value for bandwidth of MSA of test sample. In order to compute error of leave one out method for evaluation of performance of the proposed method, we can compute difference between computed value and measured value for bandwidth of MSA of any test sample and then obtained average value for all of data given in Table 5. We use leave one out method for performance testing of our proposed SVR against ANFIS with different kernel functions for SVR and different values for $\alpha$ (in this experiment we consider values $0.1,0.2, \ldots, 1$ for $\alpha$ ). Also the number of clusters is 2 . For all values of $\alpha$, average error of our method and ANFIS is shown in Table 2.

Table 2. Average error of our proposed SVR and ANFIS for calculation of bandwidths of MSA's

\begin{tabular}{ccc}
\hline Kernel & Average error for all & $\begin{array}{c}\text { Average Error } \\
\text { ANFIS }\end{array}$ \\
\hline erbf & 0.01253 & 0.1984 \\
\hline rbf & 0.32355 & 0.1984 \\
\hline Poly, $\mathrm{p}=2$ & 0.07848 & 0.1984 \\
\hline Poly, $\mathrm{p}=3$ & 0.07984 & 0.1984 \\
\hline Poly, $\mathrm{p}=4$ & 0.07495 & 0.1984 \\
\hline Linear & 0.05055 & 0.1984 \\
\hline
\end{tabular}

Also we compare our proposed method with some conventional methods [21, 23, 24 and 25]. Fig. 6 shows comparison of computed error in calculating bandwidth of MSAs with mentioned conventional methods, ANFIS and our proposed SVR method. We use simple definition for computing error as absolute value of difference between measured BW [17 and 18] and computed value using each method.

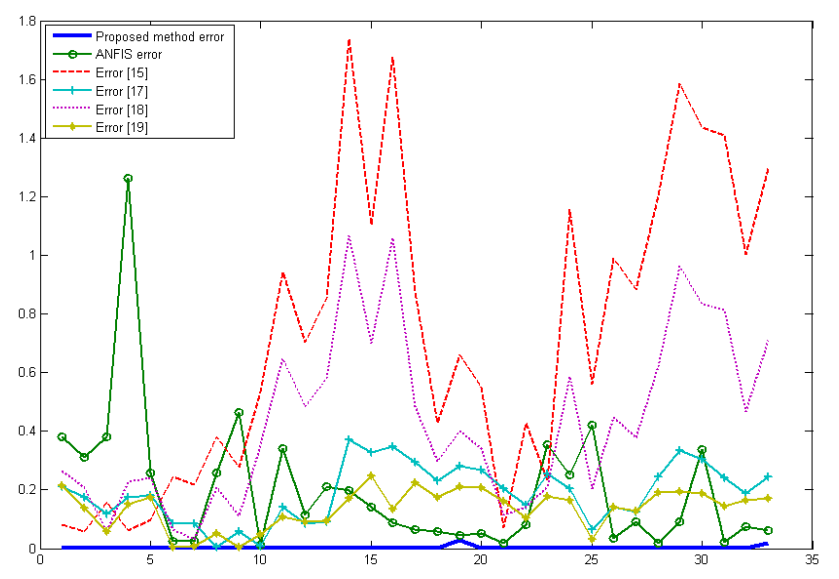

Fig. 6. Comparing error of some conventional methods and ANFIS with new SVR

Mean value of computed error for each method mentioned in Fig. 6 is as follows:

Table 3. Comparing conventional methods, ANFIS and proposed SVR for calculation of bandwidths of MSAs

\begin{tabular}{cc}
\hline Method & Error \\
\hline$[21]$ & 0.7241 \\
\hline$[23]$ & 0.1891 \\
\hline$[24]$ & 0.4337 \\
\hline$[25]$ & 0.1359 \\
\hline ANFIS & 0.1984 \\
\hline New SVR & 0.002 \\
\hline
\end{tabular}

\section{THE PSO BASED ANTENNA DESIGNING}

In the previous section we try to model bandwidth of MSA which means that we want to predict corresponding bandwidth for given input parameters correctly. For this purpose SVR and a new version of SVR is used and the result of experimentations shows the ability of presented SVR against some approaches. 
To perform a reverse action of modeling, we use particle swarm optimization (PSO) method to obtain a designing procedure.

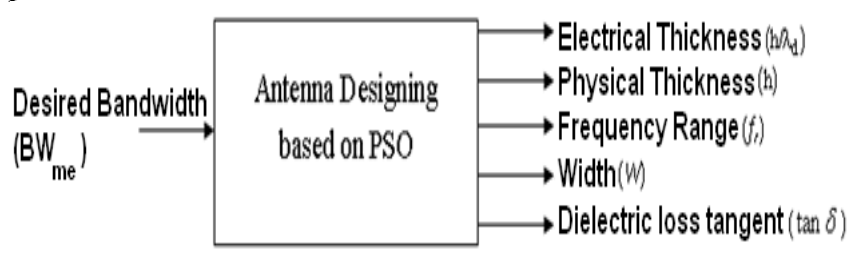

Fig. 7. Block diagram of antenna designing using PSO

As shown in Fig 1, an artificial search method can find best parameters for receiving of desired bandwidth. With search over input parameters in antenna model, desired bandwidth is obtained. Selected search method is PSO algorithm which is explained in the next sub-section. So after selection of desired bandwidth and constrains over required parameters as shown in Fig 7, PSO find these parameters based on founded model of antenna in the previous section.

In the next sub-sections, the PSO algorithm is described and then applying the search method is explained in antenna designing procedure.

\section{A. Particle Swarm Optimization}

Optimization is the process of making something better. Scientists invoke a new idea as particle swarm optimization (PSO) algorithm. The PSO was formulated by Kennedy at $1995[1,2]$. The thought process behind the algorithm was motivated by the social behavior of animals, such as bird flocking or fish schooling. Each particle moves about the cost surface with a velocity. The particles update their velocities and positions based on the local and global best solutions. We consider $\vec{x}_{i}=\left(x_{i 1}, x_{i 2}, \ldots, x_{i N}\right)$ and $\vec{v}_{i}=\left(v_{i 1}, v_{i 2}, \ldots, v_{i N}\right)$ as the position and velocity of ith particle in $\mathrm{N}$ dimensional space. The motion of an object in PSO is represented as the vector sum of present position and velocity vectors as following equation:

$\vec{x}_{i}^{k+1}=\vec{x}_{i}^{k}+\vec{v}_{i}^{k+1}$

As $\mathrm{k}$ is an iteration index number. Also in the PSO equation, $\vec{p}_{i}=\left(p_{i 1}, p_{i 2}, \ldots, p_{i N}\right)$ is the best position for ith particle in $\mathrm{N}$ dimensional space and $\vec{p}_{g}=\left(p_{g 1}, p_{g 2}, \ldots, p_{g N}\right)_{\text {would }}$ be best position for all particles. The second equation (7) comprises three vectors: inertia, competition, and cooperation. The cooperation vector $\left(c_{2}\right.$ rand $\left.\times\left(\vec{p}_{g}-\vec{x}_{i}^{k}\right)\right)$ links the current position $\left(\vec{x}_{i}^{k}\right)$ of a particle to the position of the best particle $\left(\vec{p}_{g}\right)$; it is weighted using a uniformly distributed random function. Random function helps to find of global optima in the optimization problem. Each member of the group gains knowledge of the globally best position by cooperating and communicating with all other particles. The competition vector $\left(c_{1}\right.$ rand $\times\left(\vec{p}_{i}-\vec{x}_{i}^{k}\right)$ ) links the current position of a particle to its personal best position $\vec{p}_{i}$, it is weighted using a second uniformly distributed random function. The competition factor describes the tendency of a particle to explore the vicinity of its own personal best position. Finally, the inertia vector $\left(w \vec{v}_{i}^{k}\right)$ represents the partiality of a particle to maintain its current velocity; it is weighted by a constant. PSO cleverly joins inertia, competition, and cooperation in an optimum form so that the particles swarm to the best solution: $\vec{v}_{i}^{k+1}=w \vec{v}_{i}^{k}+c_{1}$ rand $\times\left(\vec{p}_{i}-\vec{x}_{i}^{k}\right)+c_{2} \operatorname{rand} \times\left(\vec{p}_{g}-\vec{x}_{i}^{k}\right)$

The inertia weighting factor $(w)$ is important in determining the balance between global and local search capabilities. If it is too large, PSO emphasizes global searching and is slow, and if it is too small, it emphasizes local searching and gets trapped in local minima. In this paper we use the following equation for finding adaptive weighting factor:

$w=\left(w_{\text {initialize }}-w_{\text {final }}\right) \frac{\left(k_{\max }-k\right)}{k_{\max }}+w_{\text {final }}$

Where $w_{\text {initialize }}$ initialize value of weight factor and final weight is $w_{\text {final }}$, and $k_{\max }$ is maximum iteration. In the first iteration $\mathrm{k}$ is equal zero and $w$ is $w_{\text {initialize }}$ and gradually decreases to $w_{\text {final }}$. Nature of Randomness in the first iteration restricts us for following best member in the group until convergence occurs. Finally, tuning is necessary for decreasing mean square between optimal solution and founded variable.

\section{B. Designing procedure}

In this problem, we must search dimensions width (W), frequency range $\left(f_{r}\right)$, length over a ground plane with a substrate thickness (h) and dielectric loss tangent ( $\tan \delta$ ) and electrical thickness $\left(\mathrm{h} / \mathrm{\nu}_{\mathrm{d}}\right)$ with some selected constrains with user for obtaining selected bandwidth. After finding parameters, they evaluate using founded model of antenna. If desired bandwidth is satisfied and optimum parameters stand in suitable range, designing procedure is terminated. We consider antenna designing to following form,

Minimize $f\left(x_{1}, \ldots, x_{m}\right)$ Subject to

$\alpha_{i} \leq x_{i} \leq \beta_{i}, \quad i=1, \ldots, m$

$\alpha_{i}, \beta_{i}$ are boundary of constants and $x \in R^{n}$.

Here $f\left(x_{1}, \ldots, x_{m}\right)$ is evaluation function and $\alpha_{i} \leq x_{i} \leq \beta_{i}, \quad i=1, \ldots, m$ are constrains. The PSO optimizer proposes a number of particles which each particle is one proposal for $x \in R^{n}$. These particles try to minimize $f\left(x_{1}, \ldots, x_{m}\right)$ subject to constraints. If one of particle in the colony cannot find suitable result, this means that particle ( $x$ with $m$ dimension) cannot minimize $f\left(x_{1}, \ldots, x_{m}\right)$ or satisfy all constraints. So this particle should move in search space to solve problem. The particles will move in search space with $\vec{v}_{i}^{k}$ velocity which mentioned in the previous 
subsection. The following pseudo code describes our method.

Comment: PSO-based antenna designing algorithm

Comment: Define the solution space, fitness function, and population size

\section{Initialize:}

Minimum and maximum value of particles velocity in each dimension $\left[v_{\min } v_{\max }\right]$,

Maximum iteration, number of particles,

Minimum and maximum value of particles value in each dimension $\left[x_{\min } x_{\max }\right]$,

Initial and final value of Inertia weighting factor $\boldsymbol{w}$,

Determine initial value for particles $\boldsymbol{x}$.

While Iteration < Max iteration

Update inertia weighting factor $\boldsymbol{w}$

Call evaluation function; (Comment: Cost function)

Finding pbest and gbest;

Comment: pbest and gbest are best position for each and all particles respectively,

Update $\overrightarrow{\boldsymbol{v}}_{\boldsymbol{i}}^{\boldsymbol{k}+1}$ and $\overrightarrow{\boldsymbol{x}}_{\boldsymbol{i}}^{\boldsymbol{k}+1}$; (Comment: According to (7),

(6))

End While

Display founded best $\overrightarrow{\boldsymbol{x}}$.

This structure includes three main parts:

- Cost function in the PSO algorithm for solving antenna designing problem.

- Velocity weights update control.

- Tuning procedure using adaptive velocity boundaries

The PSO algorithm has been described in subsection 4.1 and here we explain matching it to this problem. $\vec{x}_{i}=\left(x_{i 1}, x_{i 2}, \ldots, x_{i m}\right)$ is ith particles with $m$ dimensions. For this problem $f\left(x_{1}, \ldots, x_{5}\right)$ has five unknown variables, so $m$ is five and $\vec{x}_{i}=\left(x_{i 1}, x_{i 2}, x_{i 3}, x_{i 4}, x_{i 5}\right)$ i.e. each particle propose one variable with $m$ dimension. Cost function determine value of each particle and search is done based on cost of all particles as explained in subsection 4.1. Next sub-section includes the PSO cost function in antenna designing problems.

\section{Cost function in the PSO algorithm}

Now problem (9) transform to a suitable function which is solved using the PSO algorithm. In general, $f\left(x_{1}, \ldots, x_{5}\right)$ is nonlinear and applied to solve (9), then we can obtain an optimization problem:

\section{$f\left(x_{1}, \ldots, x_{5}\right)=$ Desired Bandwidth- \\ Bandwidth obtained from Antenna Model}

Desired bandwidth is determined by user and it is the bandwidth that we want to find corresponding five parameters mentioned before for it. $f\left(x_{1}, \ldots, x_{5}\right)$ is calculated by subtracting desired bandwidth from bandwidth which is computed by model. As we described in detail in section 3, this model is based on SVR. Also some constraints must be applied over founded parameters which is described in the next section in details.

\section{Velocity weights update control}

In the standard PSO, the inertia weight is introduced as a decreasing function which is set to a higher value $w_{\text {initialize }}=1$ and finally it receive to $w_{\text {final }}=0.5$. Linear relation is defined per iteration according to following,

$w=\left(w_{\text {initialize }}-w_{\text {final }}\right) \frac{\left(k_{\max }-k\right)}{k_{\max }}+w_{\text {final }}$

Where the initialize value of weight factor is $w_{\text {initialize }}$ and final weight is $w_{\text {final }}, k$ is current iteration number and $k_{\text {max }}$ is maximum iteration. In the first iteration $k$ is equal zero and $w$ is $w_{\text {initialize }}$ and gradually decreases to $w_{\text {final }}$. Nature of Randomness in the first iteration restricts us for following best member in the group until convergence occurs. Finally, tuning is necessary for decreasing mean square between optimal solution and founded variable.

\section{E. Tuning procedure using adaptive velocity boundaries}

If velocity of each particle is bounded between $\left[v_{\min }, v_{\max }\right]$, controllability of PSO algorithm will be more. In the early iterations it is better we select particles with large boundary for minimum and maximum velocity. In this case particles try to fly to each area of search space. But gradually particles must move slowly which this work can performed with small velocity for $v_{\min }, v_{\max }$.

\section{EXPERIMENTAL RESULT}

Applied particles have five dimensions that they search space with various velocities. Velocity is started from high value and terminated to low value for fine searching. Physical thickness (h), frequency range $\left(f_{r}\right)$, electrical thickness $\left(h / \lambda_{d}\right)$, dimension width $(W)$ and dielectric loss tangent $(\tan \delta$ ) are variables. The antennas given in Table 5 vary in electrical thickness from 0.0065 to 0.2284 , and in physical thickness from 0.17 to $12.81 \mathrm{~mm}$, and operate over the frequency range $2.980-8.000 \mathrm{GHz}$. Their dimension width is in range 7.76-20.74 $\mathrm{mm}$ and dielectric loss tangent has values between 0.001 and 0.002 . Also these constraints can change by user definition, which means that user can define more restricted boundaries for each variable to get arbitrary result. Suppose that maximum iteration is equal to 100 . If desired bandwidth is 20, Fig. 8 and Fig.9 show error of the best particle in each iteration and obtained value for five variable. 


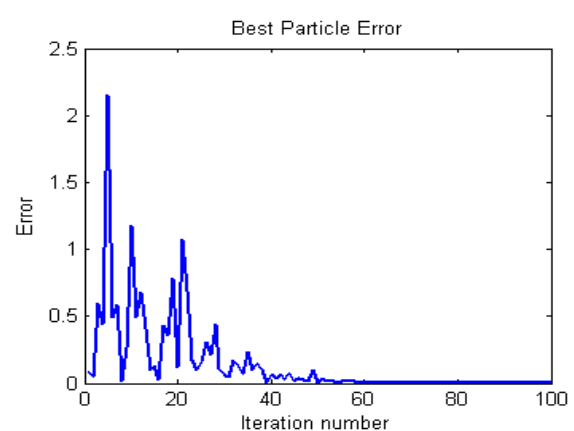

(a)

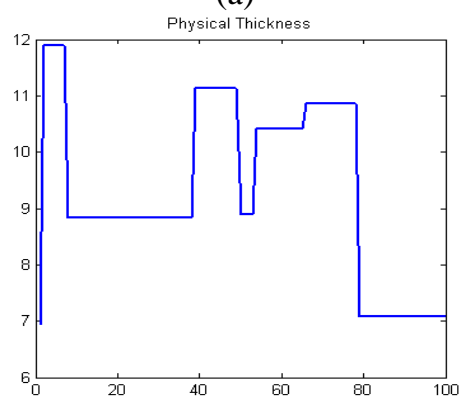

(b)

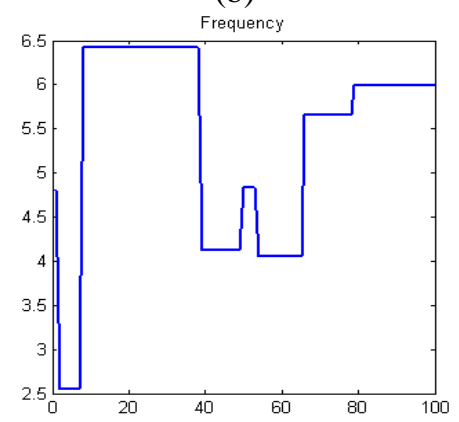

(c)

Fig. 8 (a) Absolute value of error that the best particle found, (b) Obtained value for Physical Thickness and (c) Obtained value for Frequency

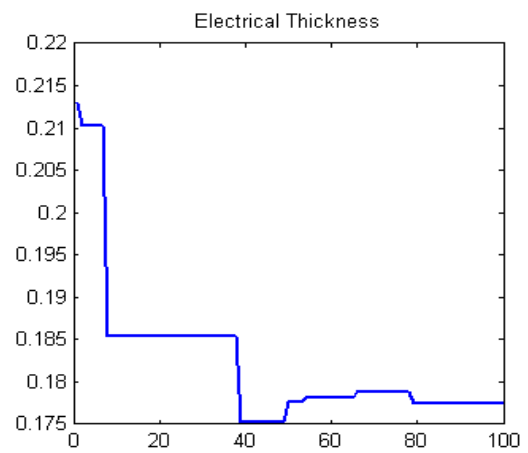

(a)

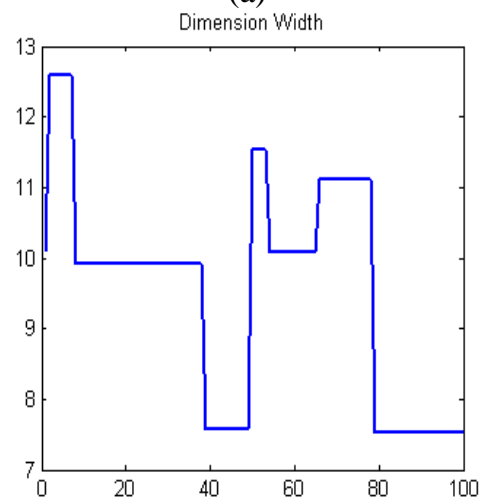

(b)

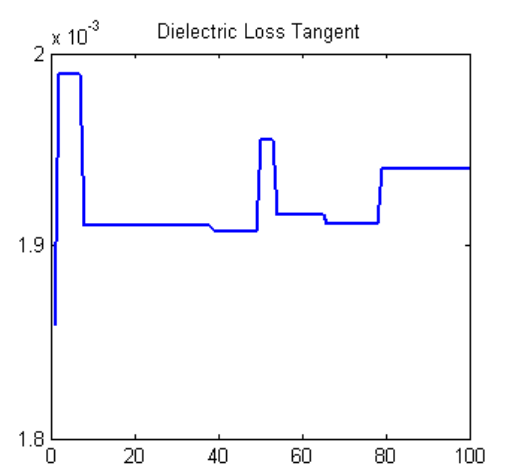

(c)

Fig. 9 (a) Obtained value for Electrical Thickness, (b) Obtained value for Dimension Width and (c) Obtained value for Dielectric Loss Tangent

In order to evaluate performance of our method, computed values of parameters by PSO in each iteration are applied in SVR for calculating corresponding bandwidth and then these bandwidth are compared to desired bandwidth (desired bandwidth is equal to 20 in this case). Fig. 10 shows the result of this comparison.

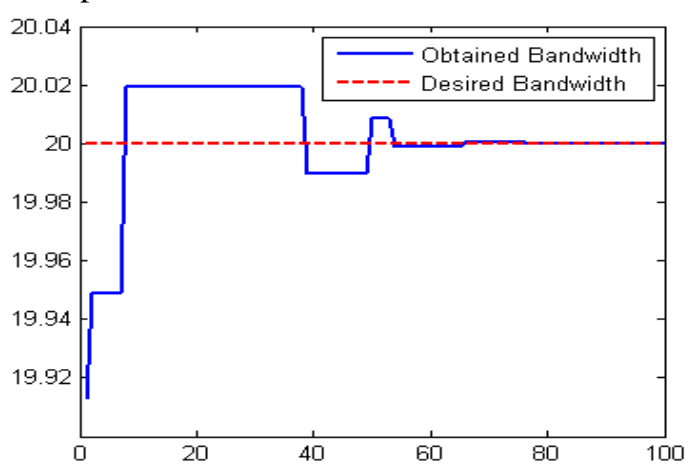

Fig. 10 Obtained bandwidth and desired bandwidth comparison

\section{A. Constraints effect}

It is mentioned before that user can select boundary of each variable more restricted. This change in constraints affects the results. For example for bandwidth 20 which we peruse it before, if we restrict constraints of variables more (correct sub-region), we get less error than the obtained error with previous constraints (a guided search). Of course for some sub-regions, it may not find any solution; for example for physical thickness, if we select limitation range between 1 to 4 , and other parameters are selected as like the previous example, divergence is occurred. So we conclude some sub-range may give divergence. Fig. 11 and Fig. 12 show error of the best particle and obtained values for each variable for following ranges.

Physical thickness has values in $8.5-9.8 \mathrm{~mm}$; frequency range is $3-4 \mathrm{GHz}$; dimension width is in range 12 to $13 \mathrm{~mm}$ and electrical thickness and dielectric loss tangent are selected like before. 


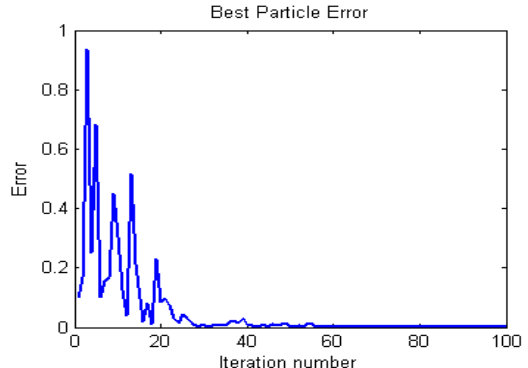

(a)

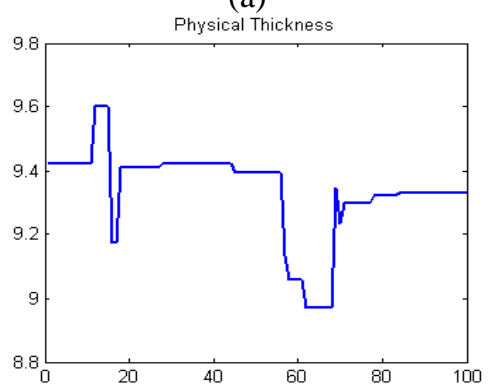

(b)

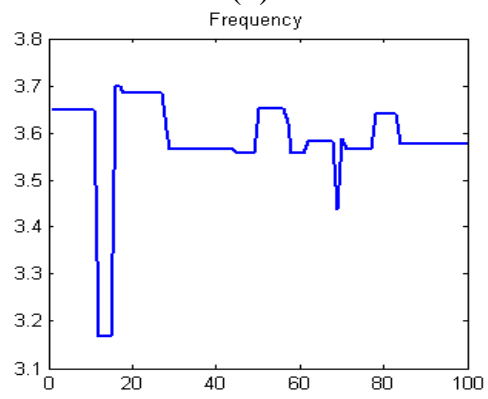

(c)

Fig. 11 (a) Absolute value of error that the best particle found, (b) Obtained value for Physical Thickness and (c) Obtained value for Frequency

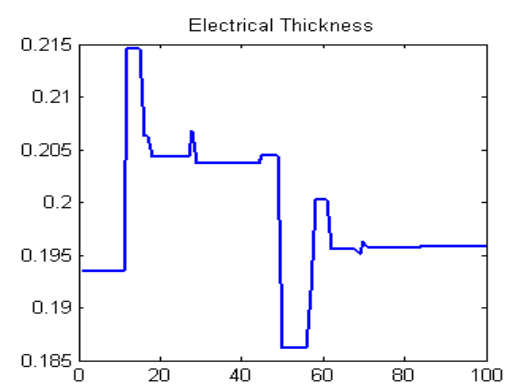

(a)

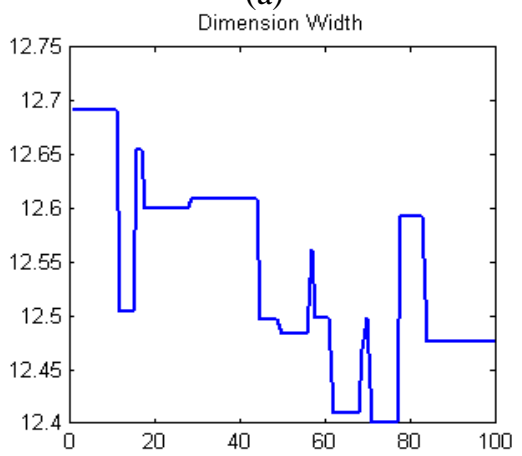

(b)

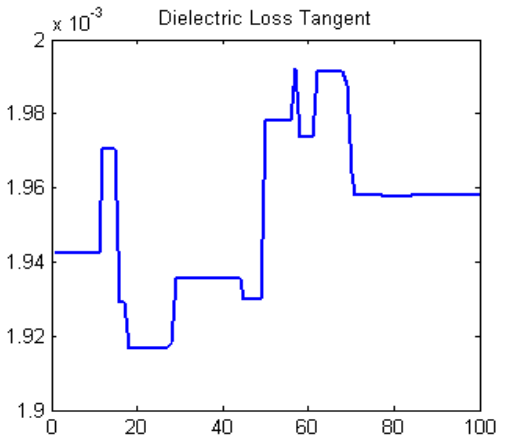

(c)

Fig. 12 (a) Obtained value for Electrical Thickness, (b) Obtained value for Dimension Width and (c) Obtained value for Dielectric Loss Tangent

As like Fig. 10, we can compare desired bandwidth with obtained bandwidths using SVR method with input parameters that PSO found. Fig. 13 shows this comparison. Also from this figure we find that, since we use guided search by more limited boundaries for five antenna parameters, we reach the desired bandwidth sooner.

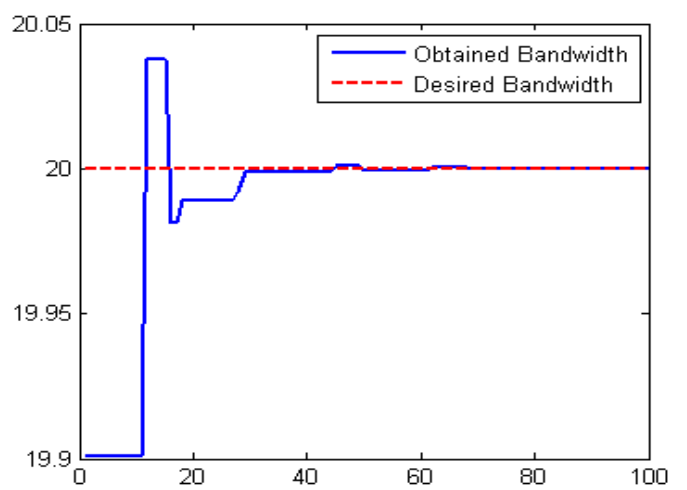

Fig. 13 Obtained bandwidth and desired bandwidth comparison

It is necessary to note that for convergence of results, range of velocity of each particle should be in -0.05 to 0.1 , otherwise PSO search process is diverged. Corresponding constraints used for MSA designing are summarized in Table 4.

Table 4. MSA parameters and their limitation ranges

\begin{tabular}{lc}
\hline MSA parameters & Range \\
\hline Physical Thickness $(h)$ & {$[0.17,12.81]$} \\
\hline Frequency $\left(f_{r}\right)$ & {$[2.98,8]$} \\
\hline Electrical thickness $\left(\mathrm{h} / \lambda_{\mathrm{d}}\right)$ & {$[0.0065,0.2248]$} \\
\hline Dimension width $(W)$ & {$[7.76,20.74]$} \\
\hline Dielectric Loss Tangent $(\tan \delta)$ & {$[0.001,0.002]$} \\
\hline
\end{tabular}

\section{CONCLUSION}

A PSO based approach for designing microstrip antennas was presented in this paper. Also in order to find a model for bandwidth calculation of MSA we used a nonlinear mathematical method namely SVR. A new version of SVR was proposed to reduce bandwidth prediction error than SVR. Thereafter designing procedure based on PSO for antenna 
designing problem was proposed in detail. The results of experimentations showed the ability of our method to find suitable parameters for antenna designing.

\section{APPENDIX}

The training and test data sets used in this paper have been obtained from previous experimental works $[17,18]$, and are given in Table 5. The 27 data sets in Table 5 were used to train the SVR. The 6 data sets, marked with an asterisk in Table 5, were used for testing. The training and test data sets used in this paper are also the same as those used for ANNs $[19,15]$ and FISs [20]. The antennas given in Table 5 vary in electrical thickness from 0.0065 to 0.2284 , and in physical thickness from 0.17 to $12.81 \mathrm{~mm}$, and operate over the frequency range $2.980-8.000 \mathrm{GHz}$.

Table 5. The measured bandwidths for electrically thin and thick rectangular

\begin{tabular}{|c|c|c|c|c|c|c|}
\hline $\begin{array}{l}\text { Patch } \\
\text { no }\end{array}$ & $\begin{array}{c}\mathrm{h} \\
(\mathrm{mm})\end{array}$ & $\begin{array}{l}\mathrm{F}_{\mathrm{r}}(\mathrm{GHZ} \\
\text { ) }\end{array}$ & $h / \lambda_{d}$ & $\begin{array}{c}W \\
(\mathrm{~mm})\end{array}$ & $\tan \delta$ & $\begin{array}{c}\text { Measured } \\
{[17,18]} \\
\text { BWme }(\%)\end{array}$ \\
\hline 1 & 0.17 & 7.740 & 0.0065 & 8.50 & 0.001 & 1.070 \\
\hline 2 & 0.79 & 3.970 & 0.0155 & 20.00 & 0.001 & 2.200 \\
\hline 3 & 0.79 & 7.730 & 0.0326 & 10.63 & 0.001 & 3.850 \\
\hline 4 & 0.79 & 3.545 & 0.0149 & 20.74 & 0.002 & 1.950 \\
\hline 5 & 1.27 & 4.600 & 0.0622 & 9.10 & 0.001 & 2.050 \\
\hline 6 & 1.57 & 5.060 & 0.0404 & 17.20 & 0.001 & 5.100 \\
\hline 7* & 1.57 & 4.805 & 0.0384 & 18.10 & 0.001 & 4.900 \\
\hline 8 & 1.63 & 6.560 & 0.0569 & 12.70 & 0.002 & 6.800 \\
\hline 9 & 1.63 & 5.600 & 0.0486 & 15.00 & 0.002 & 5.700 \\
\hline $10^{*}$ & 2.00 & 6.200 & 0.0660 & 13.37 & 0.002 & 7.700 \\
\hline 11 & 2.42 & 7.050 & 0.0908 & 11.20 & 0.002 & 10.900 \\
\hline 12 & 2.52 & 5.800 & 0.0778 & 14.03 & 0.002 & 9.300 \\
\hline 13 & 3.00 & 5.270 & 0.0833 & 15.30 & 0.002 & 10.000 \\
\hline $14 *$ & 3.00 & 7.990 & 0.1263 & 9.05 & 0.002 & 16.000 \\
\hline 15 & 3.00 & 6.570 & 0.1039 & 11.70 & 0.002 & 13.600 \\
\hline 16 & 4.76 & 5.100 & 0.1292 & 13.75 & 0.002 & 15.900 \\
\hline 17 & 3.30 & 8.000 & 0.1405 & 7.76 & 0.002 & 17.500 \\
\hline $18^{*}$ & 4.00 & 7.134 & 0.1519 & 7.90 & 0.002 & 18.200 \\
\hline 19 & 4.50 & 6.070 & 0.1454 & 9.87 & 0.002 & 17.900 \\
\hline 20 & 4.76 & 5.820 & 0.1475 & 10.00 & 0.002 & 18.000 \\
\hline 21 & 4.76 & 6.380 & 0.1617 & 8.14 & 0.002 & 19.000 \\
\hline 22 & 5.50 & 5.990 & 0.1754 & 7.90 & 0.002 & 20.000 \\
\hline 23 & 6.26 & 4.660 & 0.1553 & 12.00 & 0.002 & 18.700 \\
\hline 24 & 8.54 & 4.600 & 0.2091 & 7.83 & 0.002 & 20.900 \\
\hline 25 & 9.52 & 3.580 & 0.1814 & 12.56 & 0.002 & 20.000 \\
\hline 26 & 9.52 & 3.980 & 0.2017 & 9.74 & 0.002 & 20.600 \\
\hline $27 *$ & 9.52 & 3.900 & 0.1976 & 10.20 & 0.002 & 20.300 \\
\hline 28 & 10.00 & 3.980 & 0.2119 & 8.83 & 0.002 & 20.900 \\
\hline 29 & 11.00 & 3.900 & 0.2284 & 7.77 & 0.002 & 21.960 \\
\hline 30 & 12.00 & 3.470 & 0.2216 & 9.20 & 0.002 & 21.500 \\
\hline 31 & 12.81 & 3.200 & 0.2182 & 10.30 & 0.002 & 21.600 \\
\hline 32 & 12.81 & 2.980 & 0.2032 & 12.65 & 0.002 & 20.400 \\
\hline $33^{*}$ & 12.81 & 3.150 & 0.2148 & 10.80 & 0.002 & 21.200 \\
\hline
\end{tabular}

*Test data set

\section{REFERENCES}

[1] R. Eberhart and J. Kennedy, "A new optimizer using particle swarm theory," in Proc. 6th Int. Symposium of Micro Machine and Human Science (MHS '95), pp. 39-43, 1995.

[2] J. Kennedy and R. Eberhart, "Particle swarm optimization," in Proc. IEEE Int. Conf. Neural Networks, vol. 4, pp. 1942-1948, 1995.

[3] H-M. Feng, C-Y. Chen, F.Ye, "Evolutionary fuzzy particle swarm optimization vector quantization learning scheme in image compression", Expert Systems with Applications, vol.32, no.1, pp. 213-222, 2007
[4] M.R. Alrashidi, M.E. El-Hawary, "A survey of particle swarm optimization applications in power system operations," Electric Power Components and Systems, vol.34, no.12, pp. 1349-1357, 2006.

[5] D. W. Boeringer and D. H. Werner, "Particle Swarm Optimization versus Genetic Algorithms for Phased Array Synthesis", IEEE Trans. on Antennas and Propagation, vol.52, no.3, pp.771-779, March 2004.

[6] J. Park, K. Choi, and D. J. Allstot, "Parasitic-Aware RF Circuit Design and Optimization", IEEE Trans. on Circuits and Systems, vol.51, no.10, pp.1953-1966, Oct. 2004.

[7] D. J. Krusienski and W. K. Jenkins, "Design and Performance of Adaptive Systems Based on Structured Stochastic Optimization Strategies," IEEE Circuits and Systems Magazine, pp. 8-20, First Quarter 2005.

[8] P. Barthia, K.V.S. Rao, R.S. Tomar "Millimeter wave microstrip and printed circuit antenna" Artech House - Boston - 1991.

[9] Kiyun Han, Frances J. Harackiewiez and Seokchoo Han,"Miniaturization of microstrip patch antenna using the Sierspinski Fractal geometry", Department of Electrical \& Computer Engineering, Southern Illinois.

[10] A. K. Skrivervik, J. F. Zurcher, O. Staub and J. R. Mosig, "PCS antennas design: the challenge of miniaturization", IEEE AP Magazine, vol. 43, Aug 2001.

[11] C. A. Balanies, Antenna Theory: Analysis \& Design, John Willey \& Sons, Inc., 1997.

[12] Pozar and Schaubert, "Microstrip Antennas," Proceedings of the IEEE, vol. 80, 1992.

[13] Waterhouse, R. B., Targonski, S. D., and Kokotoff, D. M., "Design and Performance of small Printed Antennas", Trans. Antennas and Propagation, 1998, vol. 46, pp. 1629-1633.

[14] James JR, Hall PS, Handbook of microstrip antennas. IEE Electromagnetic wave series, Peregrinus, London, 1- 2(28):219-274, 1989.

[15] Gultekin S, Guney K, Sagiroglu S, "Neural networks for the calculation of bandwidth of rectangular microstrip antennas", Appl Comput Electromagn Soc J 18:46-56, 2003.

[16] Guney K, "A simple and accurate expression for the bandwidth of electrically thick rectangular microstrip antennas". Microw Opt Technol Lett 36:225-228, 2003.

[17] Kara M, "A simple technique for the calculation of bandwidth of rectangular microstrip antenna elements with various substrate thicknesses", Microw Opt Technol Lett 12:16-20, 1996.

[18] Kara M, "A novel technique to calculate the bandwidth of rectangular microstrip antenna elements with thick substrates", Microw Opt Technol Lett 12:59-64, 1996.

[19] Sagiroglu S, Guney K, Erler M, "Calculation of bandwidth for electrically thin and thick rectangular microstrip antennas with the use of multilayered perceptrons", Int J Microw Comput Aided Eng 9:277-286, 1999.

[20] Kaplan A, Guney K, Ozer S, "Fuzzy associative memories for the computation of the bandwidth of rectangular microstrip antennas with thin and thick substrates", Int J Electron 88:189-195, 2001.

[21] Bahl IJ, Bhartia P, "Microstrip antennas", Artech House, Canton, MA, 1980.

[22] Pozar DM, "Considerations for millimeter wave printed antennas", IEEE Trans Antennas Propagat 31:740-747, 1983.

[23] Carver KR, Mink JW, "Microstrip antenna technology", IEEE Trans Antennas Propagat 29:2-24, 1981.

[24] Jackson DR, Alexopoulos NG, "Simple approximate formulas for input resistance, bandwidth, and efficiency of a resonant rectangular patch", IEEE Trans Antennas Propagat 39:407-410, 1991. 